\title{
AR/VR Strategy Considerations for Academic Computing Services
}

\author{
Owen G. McGrath \\ Educational Technology Services \\ UC Berkeley, Berkeley, CA, USA, omcgrath@berkeley.edu
}

\begin{abstract}
As higher education institutions take up augmented and virtual reality (AR/VR) tools and applications, important choices and challenges arise in deciding how to approach the planning and design of infrastructure, spaces, and the services to be offered. The observations in this paper are based upon early experiences of growing AR/VR spaces and support in one university setting. The emerging services, space designs, and choice of technology and training offered are informed both by consideration of the varied $\mathrm{AR} / \mathrm{VR}$ environments being explored in the curriculum as well as a general interest in supporting AR/VR use by individuals and groups in non-curricular settings. The goals of this paper include: 1) briefly surveying commonalities and differences of AR and VR as they're being applied to teaching and learning; 2) considering service design approaches and decision points around AR/VR technologies; 3) and noting challenges for developing sustainable approaches to AR/VR support.
\end{abstract}

\section{CCS CONCEPTS}

- Applied computing• Interactive learning environments

\section{KEYWORDS}

Virtual Reality (VR), Augmented Reality (AR), Accessibility, Higher Education

\section{ACM Reference format:}

Owen G. McGrath. 2019. AR/VR Strategy Considerations for Academic Computing Services. In Proceedings of SIGUCCS '19: ACM SIGUCCS Annual Conference (SIGUCCS '19), November 03-06, 2019, New Orleans, LA, USA. ACM, New York, NY, USA, 4 pages. https://doi.org/10.1145/3347709.3347783

\footnotetext{
Permission to make digital or hard copies of all or part of this work for personal or classroom use is granted without fee provided that copies are not made or distributed for profit or commercial advantage and that copies bear this notice and the full citation on the first page. Copyrights for components of this work owned by others than ACM must be honored. Abstracting with credit is permitted. To copy otherwise, or republish, to post on servers or to redistribute to lists, requires prior specific permission and/or a fee. Request permissions from permissions@acm.org.

SIGUCCS '19, November 03-06, 2019, New Orleans, LA, USA

(c) 2019 Association for Computing Machinery.

ACM ISBN 978-1-4503-5774-6/19/11...\$15.00

https://doi.org/10.1145/3347709.3347783
}

\section{Introduction}

Across the past four decades, several dramatic transitions to new digital forms have ushered in big developmental leaps within the realm of educational technologies: digital texts, hypermedia, streaming media, and now more recently a spectrum of augmented reality (AR) and virtual reality (VR) forms and capabilities. With AR/VR's rich set of enablements, teachers, and learners have available to them a broad range of new experiential languages and modalities for exploring and adding to the stock of knowledge within and across disciplines [1]. As higher education institutions consider how best to support AR/VR for teaching and learning, opportunities and challenges arise when it comes to planning the training, infrastructure, facilities, hardware, software, and staff resources needed to deploy AR/VR services. Examining current features of the AR and VR landscape, this paper focuses on key strategic areas of focus including emerging interoperability standards, infrastructure implications, accessibility, and the curation of content.

\section{Affordances \& Applications}

\subsection{AR/VR Modes and Characteristics}

The many different uses of AR and VR are probably already uncountable, but at a high level the typical deployments in academic settings can be roughly divided into two modes: 1) vicarious experience of illusory worlds, or 2) enhanced primary experience of the so-called real world. The former category includes the many role-play games, surreal and fantasy realms but also socially impactful explorations of perspective-taking and empathy experiences [2]. For enhanced experiences of the real world, one can include augmented primary experiences of nonexistent realms that are non-fictional such as recreations of archeological sites and popular STEM titles such as the Pleito Cave archeological site [3], the International Space Station Experience [4], and the Jet Propulsion Lab's Virtual Visit to Mars [5].

It's worth noting that these two modes do not line up neatly, as might be expected, with the surface functionality and affordances of the corresponding technologies, i.e., augmented vs. immersive reality equipment. Instead, the categories are mixed. There are augmented reality experiences of illusory worlds, such as Pokémon GO that people experience in the real world. And there are also fully immersive experiences of the real 
world, such as 3D surgical brain visualizations generated from MRI scans that surgeons use before and even during surgical procedures. Finally, these two modes -- illusory and real-world experiences -- do not represent mutually exclusive options. They are more like opposite ends of a spectrum along which elements of the real and virtual world are mixed in different proportions.

\subsection{Applications in Academic Settings}

While the enabling AR/VR technologies drop in cost and become widely available as consumer commodities, the modes and functionality of AR and VR will increasingly be brought together in a variety of settings for use in teaching and learning innovations.

Commercially produced content and visualization tools are already two main areas of deployment for AR/VR in academic settings. Pre-produced content range widely across role play, virtual geographical tours science visualizations, and historical fantasy. AR/VR visualization tools extend data visualization capabilities by making them immersively explorable. For teaching and learning, these products and tools offer off-theshelf virtual experiences of places that don't exist, that exist but one cannot visit directly in real life (e.g., Mars, cortical neurons, molecular bonds), and places that no longer exist. They also provide visualizations of abstractions and data concepts that are difficult to communicate and understand.

Not surprisingly, early adoption on campuses is often found in disciplines that traditionally pursue new techniques for creating images, diagrams, or animation. In Architecture, Art History, and Chemistry, for example, 3D visualizations of building designs, physiological functions, and molecular structure bring immediate benefits to both instruction and research in the field. At UC Berkeley, architects use virtual reality simulations as a way of enhancing their understanding of how a proposed building design can optimally accommodate natural light [6]. For Art Historians, one major limiting constraint of their discipline is being able to experience paintings, sculptures, cathedrals, etc. first hand. These limitations can be overcome somewhat by virtual reality mediation that enables researchers and students to visit works of art in situ virtually even when they cannot visit such far-flung locations physically [7]. And for physical scientists whose focus is in the realm of the micro and nano, the molecular visualization tool ChimeraX from UC San Francisco recently added support for $3 \mathrm{D}$ VR output as an option, allowing researchers and students to rotate and explore molecules in virtual space while wearing a VR headset [8].

In addition to acquiring pre-produced content, higher education institutions are becoming major sites of content development as well. Custom-built AR/VR offerings, for instance, allow university libraries and museums to make their collections more accessible. Traditionally, only a fraction of a library or museum special holdings can ever be out on display. In many cases, the extension into AR/VR offerings of these collections simply extends previous digitization efforts of recent decades. If there are already digital object repositories available, they tend to lend themselves well to extension into AR/VR. For the digital patron, the leap to $3 \mathrm{D}$ experience can be transformative [9]. At UC
Berkeley, several years' worth of effort to create 3D images of Egyptian funereal objects for a web-based online museum led to a follow-on project to map that same captured date to an immersive experience of the Egyptian sarcophagi in virtual reality [10].

Development, cataloging, and curation of digital content comprise core services areas for modern libraries and museums. However, challenges that surface around AR/VR content include systems compatibility and maintainability. Depending on the application goals and chosen delivery platform, this locally created content might be produced within any number of proprietary development environments (e.g., Unity, unReal, Vizar, WebXR, ARKit, ARCore). Whether and how the various content products can be re-usable from long term archival perspective is a vexing question.

\section{Converging Services}

\subsection{Approaches to Deployment}

For the deployment of AR/VR content in instructional settings, courses usually have their needs met from a service perspective in one of three kinds of prepared settings: 1) portable AR/VR kits that can be deployed within traditional classrooms, 2) AR/VR enabled labs, where existing computer labs are re-purposed for this new technology, and 3) AR/VR labs at room scale -- that is large open spaces fully with motion sensors, layout, and equipment support for full-body activity. Across these three settings, the AR/VR solutions trade off quality for scale. In a traditional classroom or lecture hall setting, inexpensive AR/VR kits such as Google Cardboard or HoloKit can be distributed to many students assuming students own and bring sufficiently capable smart phones. In an AR/VR enabled computer lab, the quality of immersive experience improves dramatically due to the computing power at hand. However, physical movement is usually somewhat restricted as students sit within the confines of their individual lab stations. Finally, AR/VR labs at scale can offer the fullest range of motion and degrees of freedom in exploring virtual space, but now the participation level drops down to one individual at a time even while the support requirements increase [11].

For AR/VR deployments led by campus IT computing, museums, or a library, it becomes important to understand and address all three scenarios. To do so well requires a structured process for thinking about how to design such spaces to match services. Given the aims of setting up both instructional and general access $\mathrm{AR} / \mathrm{VR}$ on campus, there are many different ways to proceed. Taking an iterative approach, one can experiment with different arrangements of furniture and equipment. Rather than approaching the AR/VR as merely an extension of the existing drop-in computing facility, one should look at the range of activities to support. It tempting, for instance, to view AR/VR of as mainly just another kind of lab activity. The more powerful computing power of desktop systems still provide the best quality of rendered AR/VR experiences and are also essential for any serious AR/VR content development. The downside of this desktop lab approach is that the outcome can end up not 
matching what users want or need. For instance, the full range of AR/VR experience can offer between three and six degrees of freedom in terms of translating bodily motion into virtual world actions. Traditional computer labs where users are seated in close proximity don't always lend themselves well to the kind of body movements typical of someone wearing a headset and navigating about with hand controllers.

\subsection{Equipment Selection}

In addition to the user-oriented design factors, the selection of equipment turns out to be an interesting challenge. For the initial scaling of a facility, the equipment offered might include a full class set of tethered VR headsets in the computer lab, a small space for stand-up AR/VR experiences, and a larger AR/VR space at scale with room for multiple users and an audience. In selecting and arranging furniture, consideration needs to be given to practical matters such as warning tracks, padding, and stanchions to prevent users from colliding with surfaces or each other. VR spectator areas are a popular add-on if space allows for it. To support a culture of curiosity and sharing, displays and furniture placement can be optimized to allow for over-theshoulder visibility by an audience while still allowing for safe focus on the task.

\section{AR/VR Challenges}

\subsection{Content Curation}

At many institutions, the initial enthusiasm about developing AR/VR content has been chilled somewhat by the real-world experience of having to curate the content over time. As the technology environments proliferate and evolve, updating and adapting the existing content so that it will continue to work on the latest AR/VR software and hardware platforms can be a significant burden. These difficulties, of course, are not unprecedented for the academic computing and library communities. Maintaining expensive investments in digital assets over time [12] and managing infoglut are familiar concerns of the digital age [13].

\subsection{Interoperability}

Research and development of new AR/VR technology continue to yield more products and innovations every year. The broad array of delivery technologies to choose from includes headsets, glasses, and phones for display devices available from scores of different manufacturers. Moreover, at least a half dozen different software platforms are commonly used for the development and runtime engine aspect of $\mathrm{AR} / \mathrm{VR}$ applications. Add to this mix the varied functionality of AR/VR control devices such as hand controllers, gloves, and wearable tracking sensors quickly leads to problems of systems incompatibility. For instance, even if all hardware controllers somehow magically worked with any AR/VR application (rather than just the single system they were designed for), incompatibility issues arise even around practical implementation approaches to something as basic as input command and haptics. What counts as commands for moving, jumping, or teleporting varies dramatically across applications and controllers.

As the history of AV/IT, personal computing, and telecommunications demonstrate, the early phase of progress and innovation can quickly lead to a splintering of hardware and content development. This sort of problem is traditionally solved by developing interoperability standards. Thankfully, the AR/VR industry has now taken this important step by producing OpenXR, a comprehensive framework for device and application compatibility [14]. Several years in the making, the OpenXR provisional specification released in March 2019 provides royalty-free standards for both a common application interface and a common device interface. The standard enjoys widespread support and pledges of adoption by more dozens of companies in the member-driven Khronos Group Consortium that developed the specifications. This open-standard solution offers great potential for allowing AR/VR technology to mature and will likely result in accelerated adoption.

\subsection{Accessibility}

As with most aspects of this new medium, the accessibility questions raised around AR/VR range from negative to positive and from obvious to surprise. The new medium presents both enormous challenges and wonderful solutions for users who are differently-abled. Most immediately, AR/VR in instruction today raises many of the same accessibility issues as did streaming media (e.g., video, podcasts) when those technologies appeared on the scene. Crucial questions remain to be answered about how to provide equivalent, alternative, or accessible experiences for users who have, for instance, visual or auditory impairments. Given the importance of motion and physical control in AR/VR, there arise many equally crucial questions about accommodating various physical restrictions and limitations that users may have. The technology selection and space layout are topics where the old can inform the new. Academic libraries and computer facilities generally share a strong tradition of providing access to patrons with disabilities. Assistive technology, adjustable furniture, and careful attention to paths of travel, we're reminded, continue to be important in AR/VR. The additional needs for accommodations around controllers, sensors, and tether cabling, etc. are part of a newer area of focus across active and participatory learning spaces where the goals of independence and accommodation need careful balancing and enlightened design [15].

\section{Conclusion}

This chapter has been intended mainly as an overview of issues and opportunities that academic computing groups need to consider in defining strategy for any efforts to set up AR/VR and associated services. So far, the combination of rapid innovation and emerging standards within the marketplace stand out as key indicators that $\mathrm{AR} / \mathrm{VR}$ will soon become part of the mainstream portfolio of higher education IT service portfolios in teaching and learning. As these services mature, the next phase of service design will need to deal with crucial challenges of curating 
content over time and making AR/VR experiences accessible. Finding solutions and establishing best practices in these two areas, in particular, will be the main challenges for those in higher education who are responsible for implementing and deploying new types of AR/VR enabled teaching and learning spaces or services. And for academic technology organizations to keep up with the amazing array of new activities and experiences that the AR/VR era brings about, the service design methodologies and processes we employ will especially need an ever greater focus on delivering solutions that are cross-cutting and flexible.

\section{REFERENCES}

[1] Martín-Gutiérrez, Jorge, Carlos Efrén Mora, Beatriz Añorbe-Díaz, and Antonio González-Marrero. "Virtual technologies trends in education." EURASIA Journal of Mathematics Science and Technology Education 13, no. 2 (2017): 469-486.

[2] Herrera, Fernanda, Jeremy Bailenson, Erika Weisz, Elise Ogle, and Jamil Zaki. "Building long-term empathy: A large-scale comparison of traditional and virtual reality perspective-taking." PloS one 13, no. 10 (2018): e0204494.

[3] Robinson, David, Matthew J. Baker, Clare Bedford, Jennifer Perry, Michelle Wienhold, Julienne Bernard, Dan Reeves, Eleni Kotoula, Devlin Gandy, and James Miles. "Methodological considerations of integrating portable digital technologies in the analysis and management of complex superimposed Californian pictographs: From spectroscopy and spectral imaging to $3-\mathrm{D}$ scanning." Digital Applications in Archaeology and Cultural Heritage 2, no. 23 (2015): 166-180.

[4] Olbrich, Manuel, Holger Graf, Jens Keil, Rüdiger Gad, Steffen Bamfaste, and Frank Nicolini. "Virtual reality based space operations-a study of ESA's potential for VR based training and simulation." In International Conference on Virtual, Augmented and Mixed Reality, pp. 438-451. Springer, Cham, 2018.

[5] Law, E. S., \& Day, B. H. (2018). STEM Engagement with NASA's Solar System Treks Portals for Lunar and Planetary Mapping and Modeling.

[6] Santos, Luis, António Leitão, and Luisa Caldas. "A comparison of two lightredirecting fenestration systems using a modified modeling technique for Radiance 3-phase method simulations." Solar Energy 161 (2018): 47-63.

[7] Klinke, Harald, Liska Surkemper, and Justin Underhill. "Creating New Spaces in Art History." International Journal for Digital Art History: Issue 3, 2018 : Digital Space and Architecture 3 (2019): 9.

[8] Goddard, Thomas D., Conrad C. Huang, Elaine C. Meng, Eric F. Pettersen, Gregory S. Couch, John H. Morris, and Thomas E. Ferrin. "UCSF ChimeraX: Meeting modern challenges in visualization and analysis." Protein Science 27, no. 1 (2018): 14-25.

[9] Lucarelli, R. (2016). "Images of eternity in 3D: The visualization of ancient egyptian coffins through photogrammetry". In Monica Berti and Franziska Naether (Eds.), Altertumswissenschaften in a Digital Age: Egyptology, Papyrology and Beyond; Proceedings of a Conference and Workshop in Leipzig, November 4-6. Leipzig, Beitrag 24

[10] Bruno, Fabio, Stefano Bruno, Giovanna De Sensi, Maria-Laura Luchi, Stefania Mancuso, and Maurizio Muzzupappa. "From 3D reconstruction to virtual reality: A complete methodology for digital archaeological exhibition." Journal of Cultural Heritage 11, no. 1 (2010): 42-49.

[11] Akçayır, Murat, and Gökçe Akçayır. "Advantages and challenges associated with augmented reality for education: A systematic review of the literature." Educational Research Review 20 (2017): 1-11.

[12] Lynch, Clifford A. "Institutional repositories: essential infrastructure for scholarship in the digital age." portal: Libraries and the Academy 3, no. 2 (2003): 327-336.

[13] McGrath, Owen G. "Organizing new interactions: internet portals in the instructional computer lab." In Proceedings of the 29th annual ACM SIGUCCS conference on User services, pp. 101-105. ACM, 2001.

[14] Khronos.org. OpenXR Architecture. Accessed: June 10, 2019. [Online]. Available: https://www.khronos.org/openxr

[15] McGrath, Owen G. "Making a Makerspace: Designing User Services to Serve Designing Users." In Proceedings of the 2016 ACM on SIGUCCS Annual Conference, pp. 95-98. ACM, 2016. 